\title{
Análisis espacial de la inclusión financiera y su relación con el nivel de pobreza en los municipios mexicanos Pablo Pérez Akaki*
}

Universidad Nacional Autónoma de México, Facultad de Estudios Superiores, Acatlán María del Rocío Fonseca Soto

Universidad Nacional Autónoma de México, Facultad de Estudios Superiores, Acatlán

(Recibido 02 de Diciembre 2015, aceptado 17 de Junio 2016)

\begin{abstract}
Resumen
El objetivo de este trabajo es conocer la importancia que tiene la inclusión financiera y su relación estadística espacial con la pobreza a nivel municipal en México en el año 2010. Para ello, se propone que mediante el uso de las técnicas de análisis espacial econométrico se evalúe la relación entre estos conceptos en el caso de los municipios de México, incluyendo un conjunto de variables de control a nivel municipal. En el ejercicio de análisis se encontró que existe una relación importante entre inclusión financiera y pobreza a nivel municipal, aunque depende de la definición usada de inclusión. Los ejercicios empíricos desarrollados corresponden al año 2010, por lo que los resultados no son generalizables a las fechas actuales, aunque dan evidencia de esa relación en un pasado reciente. La originalidad del trabajo radica en que el uso de la metodología para el análisis de estos casos no se había dado con anterioridad, lo cual destaca la importancia de las vecindades territoriales y de la importancia entonces del espacio en el diseño de soluciones a los problemas del desarrollo. El trabajo concluye que existe una relación causal importante y positiva entre inclusión financiera y la pobreza municipal, la cual es más robusta cuando incorpora las vecindades espaciales.
\end{abstract}

Clasificación JEL: R51, 016.

Palabras clave: Inclusión Financiera, México, Desarrollo Económico, Municipios, Pobreza.

\section{Spatial Analysis of Financial Inclusion and the Relation with Poverty Level in Mexican Municipalities}

\begin{abstract}
The aim of this study was to determine the importance of financial inclusion and spatial statistics regarding poverty at the municipal level in Mexico in 2010. To this end, it is proposed the use of techniques of spatial econometric analysis to evaluate the relationship between these concepts in the case of the municipalities of Mexico, including a set of control variables at the municipal level. The results of estimation of econometric models found that there is an important relationship between financial inclusion and poverty at the municipal level, although it depends on the definition used for financial inclusion. The empirical exercises were developed for the year 2010, so the results are not generalizable to the current dates, but provide evidence of that relationship in the recent past. The originality of this work is that the use of the methodology for the analysis of these cases had not previously given, highlighting the importance of territorial neighborhoods and the importance of space in designing solutions to problems of development. The paper concludes that there is a significant and positive causal relationship between financial inclusion and municipal poverty, which is more robust when space incorporates neighborhoods.
\end{abstract}

JEL Classification: R51,016.

Key Words: Financial Inclusion, Mexico, Economic Development, Municipalities, Poverty.

* Alcanfores y San Juan Totoltepec SN. Col. Santa Cruz Acatlán, C.P. 53150. Naucalpan, Estado de México. Tel. (55)56231559. Correo Electrónico:ppablo@apolo.acatlan.unam.mx 


\section{El sector financiero y su importancia en el crecimiento económico}

Uno de los objetivos de la política económica es favorecer el aumento de la producción de bienes y de la prestación de servicios en un país y la adquisición de los mismos, es decir, el crecimiento económico, ya que éste, directa e indirectamente, se relaciona con el bienestar de la población. Los bienes y servicios se producen u ofrecen a partir de la combinación de los factores de producción por lo que el capital, el trabajo, las habilidades empresariales y la tierra se convierten en elementos fundamentales de análisis para modelar el crecimiento económico. Adicionalmente, se ha identificado que influyen en el crecimiento económico de un país la estabilidad política, las instituciones, las leyes, el gobierno, las relaciones con otros países y, del que nos ocupamos en este trabajo, el sistema financiero (La Porta, 1997; Khalifa, 2002; Honohan, 2004 y Ang, 2008 entre otros).

De acuerdo con Levine (1997) las funciones de los sistemas financieros que impulsan el mejoramiento de las condiciones de vida de la sociedad son:

- Facilitar el manejo del riesgo: el sistema financiero contribuye a disminuir el riesgo de liquidez mediante mecanismos que facilitan que los ahorradores puedan disponer de sus activos financieros en el momento que lo requieran y que las empresas tengan acceso permanente a fondos para inversión. Además, al ofrecer diversas opciones para inversión, también disminuye el riesgo para los ahorradores e inversionistas. Señala que los sistemas financieros que facilitan la diversificación del riesgo pueden acelerar el cambio tecnológico y el crecimiento económico.

- Asignar recursos: los intermediarios financieros están especializados en la obtención de información completa y pertinente acerca de los proyectos de inversión a fin de seleccionar los más rentables y destinar los recursos para financiarlos. Los intermediarios financieros identifican las mejores tecnologías de producción y servicio y con ello impulsan la innovación tecnológica en la industria, dado el vínculo con otros sectores, pueden generar efectos de derrama.

- Monitorear a los administradores y ejercer el control corporativo: se refiere a las actividades de seguimiento de los proyectos financiados por los intermediarios financieros y que a veces se denota como un acuerdo de "supervisión delegada".

- Movilizar los ahorros, evaluar los riesgos y asignar los recursos disponibles: esto implica el reunir ahorros de distintas personas y destinarlos a los mejores proyectos productivos. Al realizar esta actividad los intermediarios financieros reducen los costos en los que pudieran incurrir tanto los ahorradores, en la búsqueda de información y en la evaluación para la toma de decisiones, como las empresas, en la consecución de recursos de diferentes inversionistas.

- Facilitar el intercambio: tiene que ver con la eficiencia de los sistemas de pago que favorecen las transacciones entre agentes económicos.

La contribución del sistema financiero al crecimiento económico probablemente sería mayor cuanto más amplio fuera el conjunto de instrumentos financieros disponibles e instituciones financieras en operación y mientras más se apegaran a las reglas de la libre competencia (Goldsmith, 1963). Recientemente se han realizado varios estudios empíricos que permitieron identificar una relación positiva entre el sistema financiero y el crecimiento 
económico, entre los que se encuentran King y Levine (1993), Beck, DemirgücKunt y Levine (2004) y Rodríguez y López (2009), entre los más destacados.

Igualmente, se ha encontrado evidencia que respalda que el mayor desarrollo de un sistema financiero es consecuencia de una mayor riqueza en los países, lo cual acelera el crecimiento económico pues facilita el financiamiento para la creación de nuevas empresas y el financiamiento externo de las ya existentes (Demirgürc-Kunt y Levine, 2001:11). Si bien la literatura teórica y empírica citada anteriormente ha estudiado la relación entre desarrollo del sector financiero y crecimiento económico, no se ha profundizado en la relación entre sector financiero y desarrollo de la sociedad. Por ello en este trabajo, se busca avanzar en este vacío de información como aproximación al desarrollo económico de una sociedad, el nivel de pobreza, que puede considerarse una variable inversa.

En un documento de trabajo del Banco Mundial, Claessens y Feijen (2006) señalan haber encontrado una relación negativa entre el crecimiento del PIB per cápita y la pobreza, la que también está relacionada con la educación, la salud y la igualdad de género. Cuando el PIB per cápita crece como resultado del desarrollo financiero los hogares se benefician de mayores ingresos y pueden consumir e invertir más, lo que se reflejará en el bienestar del hogar en términos de nutrición, cuidado de la salud y mayor educación. En la investigación también se identifican los canales a través de los cuales un sistema financiero eficiente puede incidir en el crecimiento económico, como se muestra en la Figura 1.

Si el sistema financiero de un país influye positivamente en su crecimiento económico, entonces es fundamental asegurar que toda la población tenga acceso a los servicios y productos financieros disponibles. Con esta perspectiva, una corriente de pensamiento surgida hace poco tiempo que ha cobrado auge entre los organismos públicos mundiales es la inclusión financiera, la cual ha sido impulsada fuertemente también por el gobierno de México y el sistema financiero nacional.

Sin embargo, existen dudas sobre este concepto y su importancia para resolver los problemas de rezago económico en algunas regiones del país, las cuales se originan a partir de los siguientes hechos: por una parte se ha señalado que una de las funciones más importantes del sistema financiero es asignar de forma eficiente los recursos excedentes hacia donde hacen falta, pero el desempeño de nuestro sistema financiero en relación con esta función ha sido deficiente (Avalos y Trillo, 2006: 11), en parte debido a que en muchos casos no se puede asignar los recursos porque quienes los necesitan no cumplen con los requisitos para obtener un crédito, lo que algunos identifican como una segmentación de mercado (Rivera y Rodríguez, 2007: 42). Otro motivo por el cual los que necesitan recursos no los reciben, por lo menos de instituciones financieras como los bancos, consiste en que en la actualidad dichas instituciones prefieren invertir en valores gubernamentales que en proyectos productivos. Por otra parte, los esfuerzos para hacer que los servicios y productos financieros estén disponibles para actividades productivas, en un horizonte de largo plazo ha venido en deterioro (Rivera y Rodríguez, 2007: 50) y aunque recientemente el acceso a los servicios financieros se ha ampliado por medio de los corresponsales bancarios, los efectos identificados eran modestos (Peña y Vázquez, 2012). 
Una estrategia adicional para ofrecer servicios y productos financieros específicamente a la población con bajos ingresos, o que trabajan en el sector informal, o que viven en zonas rurales consiste en la creación de instituciones de microfinanzas, de las que se ha dicho además que pueden contribuir a la disminución de la pobreza; aunque este objetivo adicional no ha sido notorio aún en nuestro país. Si bien se han mencionado algunos obstáculos para mejorar el desempeño del sistema financiero mexicano, deben mantenerse los avances logrados hasta el momento y diseñarse estrategias adicionales para lograr el objetivo de aumentar el número de personas con acceso a los productos y servicios financieros a fin de elevar su nivel de bienestar.

\section{La inclusión financiera en México: su importancia y su medición.}

El nivel de los servicios financieros existentes en un país puede medirse a través de distintos indicadores, por ejemplo, la relación crédito privado/producto interno bruto o la relación M2/producto interno bruto. El nivel de los servicios financieros medido así, por alguno de estos indicadores, se conoce como profundización financiera.

Recientemente, con un impulso de los organismos financieros internacionales, se ha popularizado el concepto de inclusión financiera, asociado con un conjunto de indicadores que tratan de medir más ampliamente la profundidad financiera de las economías. De acuerdo con el Center for Financial Inclusion, este concepto se refiere a una situación en la que las personas tienen acceso a un conjunto de instrumentos financieros de calidad, provistos a precios accesibles, en una manera conveniente, con un respeto por su dignidad de clientes (CFI, 2009a). Estos instrumentos y servicios de calidad comprenden medios de pago, ahorros, créditos y seguros (CFI, 2009b).

Figura 1. Desarrollo financiero y los canales de crecimiento del PIB Per Cápita.

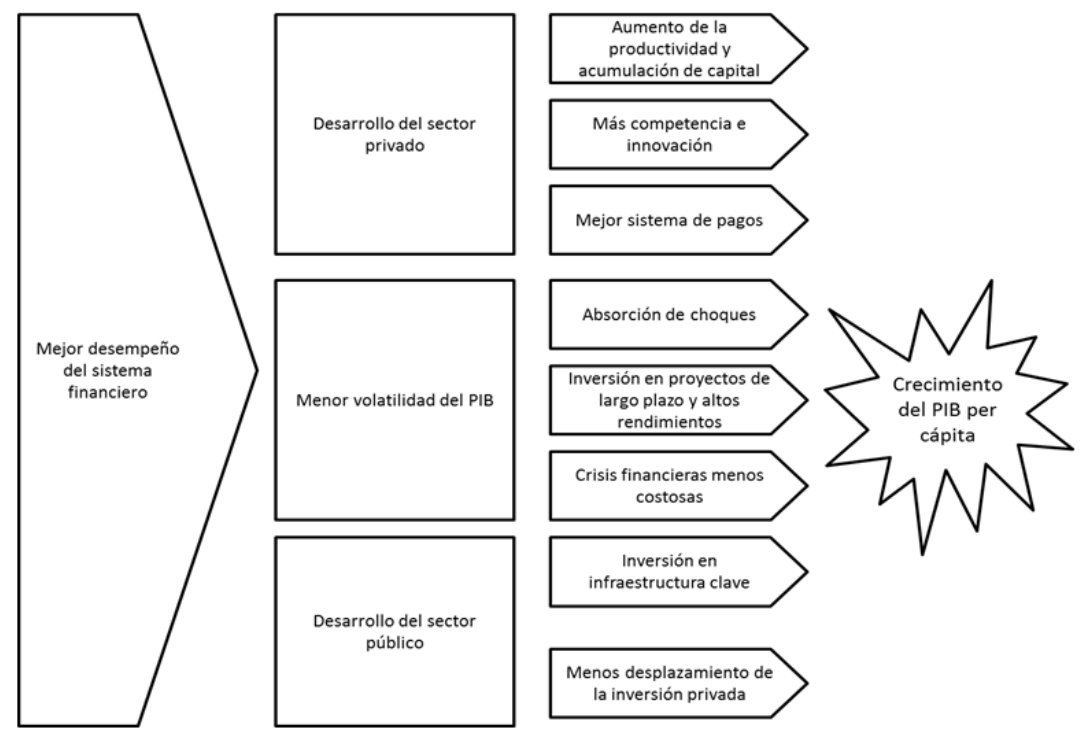

Fuente: Claessens y Feijen, 2006. 
Demirguc-Kunt y Klapper (2012) publicaron los resultados del primer análisis de la Global Financial Inclusion Database (Global Findex). Este fue un proyecto financiado por la Fundación Bill y Melinda Gates con el objetivo de conocer el uso que hace la gente de los servicios financieros. Para ello construyeron indicadores con datos obtenidos en encuestas aplicadas a más de 150,000 adultos durante 2011, a lo largo de 148 países de ingresos altos, medios y bajos. ${ }^{1}$ Los indicadores fueron clasificados en cuatro conjuntos: cuentas formales, ahorro, crédito y seguros. El conjunto de indicadores de cuentas formales permite medir principalmente la frecuencia y la forma de acceder a las cuentas, ${ }^{2}$ así como conocer el tipo de cuenta, es decir, si la cuenta es personal o de negocios, si es de nómina o para recibir apoyos del gobierno o remesas; el conjunto de indicadores relacionados con el ahorro permite conocer el número de personas que tienen una cuenta de ahorro en alguna institución financiera formal; los indicadores de crédito se enfocan en las fuentes de financiamiento: formales o informales, en el destino del crédito y en el uso de tarjetas de crédito; mientras que los indicadores relacionados con los seguros se refieren a la protección para el cuidado de la salud y para la agricultura, principalmente, con que cuenta la población.

En el Gráfica 1 se muestra el valor que tiene el indicador de porcentaje de población adulta con cuenta en una institución financiera formal (IFF) para algunos países de Latinoamérica y el Caribe. Los datos revelan una condición de valores bajos para México en algunos de los indicadores relacionados con la inclusión financiera, muy por detrás de países comparables en tamaño de su economía, como Argentina y Brasil e incluso de otros más pequeños como Costa Rica.

\footnotetext{
1 Un adulto es una persona con 15 o más años de edad.

2 Las autoras definen cuentas formales como aquéllas que se realizan en una institución financiera formal tal como un banco, una unión de crédito, cooperativa, oficina postal o institución microfinanciera.
} 
Gráfica 1. Proporción de la población adulta con cuenta en una institución financiera formal (IFF), con cuenta de ahorro y con tarjeta de crédito.

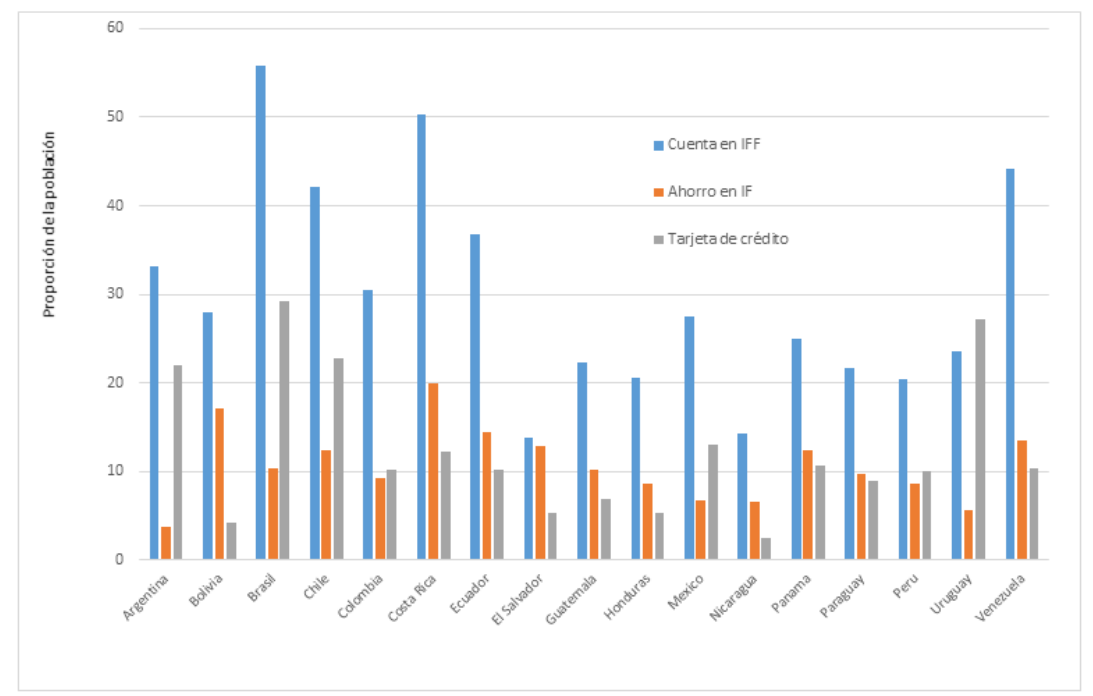

Fuente: Elaboración propia con datos de Global Financial Inclusion Database, disponibles en http://databank.worldbank.org/data/views/variableselection/selectvariables.aspx?source=global-findex

En el Gráfica 2 se muestra el porcentaje de población adulta que contaba con algún préstamo en el momento de la encuesta, así como la fuente del crédito, que puede ser formal o informal. En el caso de México se puede observar que la mayoría de las personas con crédito lo ha conseguido de fuentes informales destacando, como en casi todos los países incluidos, que la familia y los amigos son la principal fuente de financiamiento.

En los últimos años, dada esta evidencia empírica, el gobierno de México emprendió una estrategia para aumentar la inclusión financiera de su población, la cual comenzó cuando México, por medio de la Comisión Nacional Bancaria y de Valores (CNBV), organismo dependiente de la Secretaría de Hacienda y Crédito Público, adquirió su membresía a la Alianza para la Inclusión Financiera, institución que cuenta con el respaldo del Center for Financial Inclusion. Los pasos que se dieron para impulsar esta estrategia fueron, en primer lugar, establecer una definición de inclusión financiera apropiada para nuestro país, la cual quedó contenida en el Acuerdo por el que se crea el Consejo Nacional de Inclusión Financiera, publicado el 3 de octubre de 2011 en el Diario Oficial de la Federación, donde se menciona que la inclusión financiera "comprende el acceso y uso de servicios financieros bajo una regulación apropiada que garantice esquemas de protección al consumidor y promueva la educación financiera para mejorar las capacidades financieras de todos los segmentos de la población" (Diario Oficial de la Federación, 03/10/2011).

Paralelamente, a partir de un diagnóstico elaborado por el Center for Financial Inclusion (2009b) se propuso un conjunto de indicadores para medir 
el grado o nivel de inclusión financiera para México. El cálculo de los valores de los indicadores lo realizó la Comisión Nacional Bancaria y de Valores a partir de la información de los intermediarios financieros. Como resultado de este trabajo se han publicado seis reportes de inclusión financiera de 2009 a 2015 y en 2012 se levantó la primera Encuesta de Inclusión Financiera (Instituto Nacional de Estadística y Geografía, 2012) representativa a nivel nacional.

Gráfica 2. Población adulta con algún préstamo, según fuente.

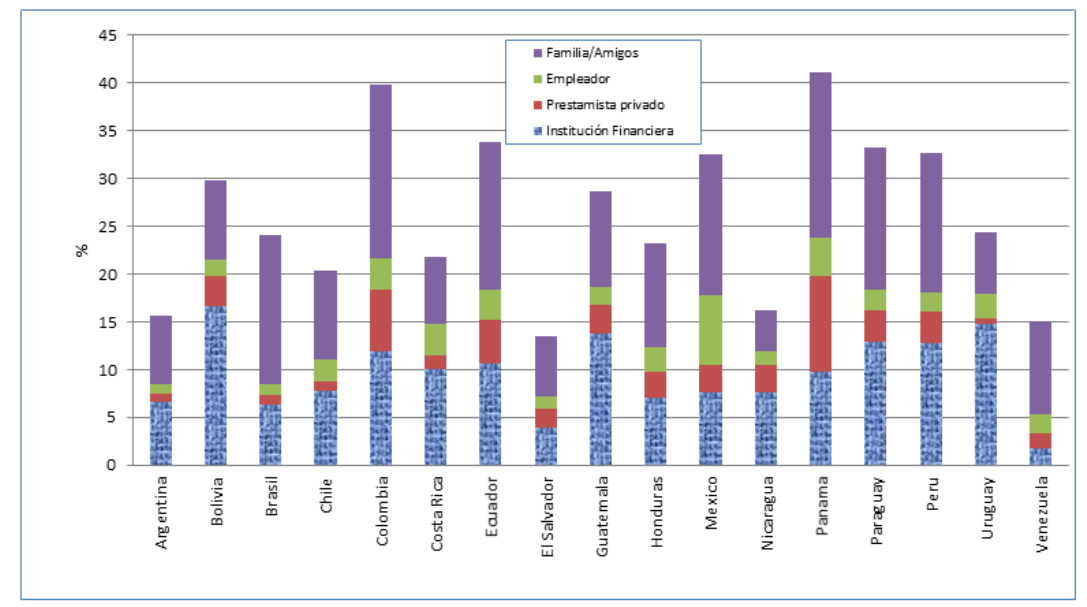

Fuente: Elaboración propia con datos de Global Financial Inclusion Database, disponibles en http://databank.worldbank.org/data/views/variableselection/selectvariables. aspx?source=global-findex

Los resultados encontrados para México no fueron muy alentadores, pues se encontraron importantes diferencias entre entidades y municipios respecto a su nivel de inclusión financiera. Con base en los datos de la ENIF 2012 se presenta el Cuadro 1 que compila los resultados más importantes de este instrumento. Los argumentos más importantes a los resultados encontrados, que impiden que el alcance de la inclusión financiera sea más amplio, fueron:

1. Más del $64 \%$ de los $70^{\prime} 382,459$ adultos no ahorra formalmente, principalmente porque sus ingresos son insuficientes o variables.

2. Aproximadamente el $77 \%$ no cuenta con algún tipo de seguro, básicamente porque son muy caros.

3. Alrededor del $60 \%$ no utiliza sucursales bancarias primordialmente porque no tiene una cuenta o tarjeta, mientras que el $62 \%$ no utiliza cajeros automáticos.

4. Es notable también que al $16 \%$ de la población que habita en localidades con menos de 15,000 habitantes le toma más de una hora trasladarse a una sucursal bancaria. Para el $62 \%$ de los adultos el costo de ir a una sucursal bancaria se encuentra entre uno y cincuenta pesos.

5. Menos del $30 \%$ de los adultos recurre al menos a un mecanismo de crédito formal, mientras que el $34 \%$ recurre al crédito informal, principalmente al otorgado por la familia. 
50 Nueva Época REMEF (The Mexican Journal of Economics and Finance)

6. El uso de los seguros es muy bajo pues apenas alrededor del $20 \%$ cuenta con uno.

Cuadro 1. Resultados relevantes de la Encuesta Nacional de Inclusión Financiera 2012 según tamaño de localidad.

\begin{tabular}{|c|c|c|c|}
\hline \multirow{2}{*}{ Variable } & \multicolumn{2}{|c|}{ Tamaño de localid ad } & \multirow[b]{2}{*}{ Nacional } \\
\hline & $\begin{array}{c}\text { Menos de 15,000 } \\
\text { habitantes }\end{array}$ & $\begin{array}{l}\text { Más de } 15,000 \\
\text { h abitantes }\end{array}$ & \\
\hline Nivel educativo predominante (\%) & Secundaria (28) & Secundaria (29) & Secundaria (29) \\
\hline $\begin{array}{l}\text { Fuente de recursos más utilizada para } \\
\text { pagar alguna emergencia (\%) }\end{array}$ & $\begin{array}{l}\text { Préstamo entre } \\
\text { particulares (74) }\end{array}$ & $\begin{array}{l}\text { Préstamo entre } \\
\text { particulares (64) }\end{array}$ & $\begin{array}{l}\text { Préstamo entre } \\
\text { particulares (67) }\end{array}$ \\
\hline $\begin{array}{l}\text { Porcentaje de adultos con al menos un } \\
\text { producto de ahorro formal }\end{array}$ & 22 & 42 & 36 \\
\hline Porcentaje de usuarios de ahorro informal & 42 & 45 & 44 \\
\hline $\begin{array}{l}\text { Mecanismo más utilizado por los } \\
\text { ahorradores informales (\%) }\end{array}$ & En casa $(31)$ & En casa $(27)$ & En casa $(28)$ \\
\hline $\begin{array}{l}\text { Porcentaje de adultos que usa al menos un } \\
\text { mecanismo de crédito formal }\end{array}$ & 17 & 33 & 27 \\
\hline $\begin{array}{l}\text { Porcentaje de adultos que usa al menos un } \\
\text { mecanismo de crédito informal }\end{array}$ & 35 & 33 & 34 \\
\hline $\begin{array}{l}\text { Mecanismo de crédito informal más } \\
\text { utilizado (\%) }\end{array}$ & Familia (20) & Familia (19) & Familia (19) \\
\hline \multirow{2}{*}{$\begin{array}{l}\text { Principales destinos del crédito informal } \\
\text { (\%) }\end{array}$} & $\begin{array}{c}\text { Gastos personales } \\
\text { (34) }\end{array}$ & $\begin{array}{l}\text { Gastos personales } \\
\text { (33) }\end{array}$ & $\begin{array}{c}\text { Gastos personales } \\
\text { (33) }\end{array}$ \\
\hline & $\begin{array}{l}\text { Educación o salud } \\
\text { (32) }\end{array}$ & $\begin{array}{l}\text { Educación o salud } \\
\text { (26) }\end{array}$ & $\begin{array}{c}\text { Educación o salud } \\
\text { (28) }\end{array}$ \\
\hline $\begin{array}{l}\text { Porcentaje de usuarios de seguros } \\
\text { privados }\end{array}$ & 11 & 28 & 22 \\
\hline $\begin{array}{l}\text { Porcentaje de usuarios de cuentas de } \\
\text { ahorro para el retiro }\end{array}$ & 14 & 35 & 28 \\
\hline $\begin{array}{l}\text { Porcentaje de adultos que reciben remesas } \\
\text { como parte de sus ingresos }\end{array}$ & 11 & 8 & 9 \\
\hline \multirow[t]{2}{*}{$\begin{array}{l}\text { Principales operaciones realizadas en } \\
\text { sucursales bancarias (\%) }\end{array}$} & $\begin{array}{l}\text { Retiro de efectivo } \\
\text { (58) }\end{array}$ & $\begin{array}{l}\text { Retiro de efectivo } \\
\text { (55) }\end{array}$ & $\begin{array}{c}\text { Retiro de efectivo } \\
\text { (56) }\end{array}$ \\
\hline & Depósitos (39) & Depósitos (53) & Depósitos (49) \\
\hline $\begin{array}{l}\text { Porcentaje de adul tos a quienes les toma } \\
\text { más de una hora de tiempo trasladarse a } \\
\text { una sucursal bancaria }\end{array}$ & 16 & 2 & 5 \\
\hline
\end{tabular}

Notas: Productos de ahorro formal: nómina, ahorro, cheques, plazo, fondo de inversión. Mecanismos de ahorro informal: en casa, tanda, préstamo, caja de ahorro y familia. Mecanismos de crédito informal: Familia, amigos, caja de ahorro entre amigos o conocidos, casa de empeño.

Fuente: Elaboración propia con base en Encuesta Nacional de Inclusión Financiera.

Si a lo anterior le agregamos que $70 \%$ de los trabajadores mexicanos recibe menos de $\$ 5,000$ pesos mensuales como ingreso por su trabajo y que $97.7 \%$ 
era empleado, obrero, jornalero, peón, trabajador por su cuenta o sin pago, entonces podría decirse que una parte significativa del total de estas personas por su nivel de ingresos está excluida de los servicios financieros o es muy limitada su participación. Más del cincuenta por ciento de la población adulta no usa los servicios financieros, mayormente en localidades menores a 15,000 habitantes, lo que sugiere una asociación positiva entre urbanidad e inclusión financiera, ${ }^{3}$ así como una relación negativa indirecta entre inclusión financiera y pobreza, ${ }^{4}$ tal como se muestra en el Gráfica 3. Sin embargo, es claro que no puede establecerse una relación lineal inversa estricta entre pobreza e inclusión financiera, pues existen numerosos municipios en donde la pobreza es baja y no se presenta alta inclusión financiera. Esto sugiere que existen otros factores que la explican. Uno de estos factores es la geografía, la cual de manera indirecta puede cuantificarse por medio de la influencia que sobre un municipio tienen los municipios vecinos, es decir, los efectos espaciales de la inclusión financiera y el desarrollo económico propio de cada municipio.

Gráfica 3. Inclusión financiera, urbanidad y pobreza en los municipios de México.
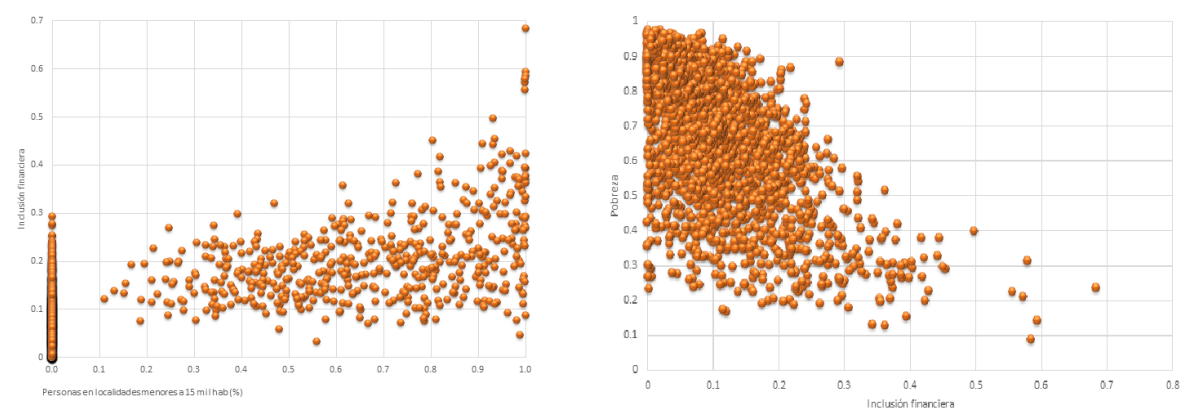

Fuente: Elaboración propia con datos del RIF 4, Censo de Población y Vivienda 2010 y CONEVAL.

En la literatura sobre economía regional se señala que si los datos para el análisis son de corte transversal, es necesario incluir variables relacionadas con el espacio geográfico para identificar si existe alguna relación entre unidades geográficas cercanas pues "todo está relacionado con todo lo demás, pero las cosas cercanas están más relacionadas entre sí que las cosas distantes" (Tobler, 1970). Un argumento importante en este sentido es el reconocer que no se trata

3 El criterio de urbanidad utilizado corresponde a la proporción de personas en el municipio que habitan en localidades de mayor tamaño a 15 mil habitantes, de acuerdo con el Censo de Población y Vivienda del 2010.

4 La pobreza se identificó a partir de la medición municipal del 2010 del Consejo Nacional de Evaluación de la Política de Desarrollo Social (CONEVAL) y corresponde a la proporción de personas del municipio en situación de pobreza (moderada y extrema). La definición usada es "Una persona se considera en situación de pobreza cuando no tiene garantizado el ejercicio de al menos uno de sus derechos para el desarrollo social, y sus ingresos son insuficientes para adquirir los bienes y servicios que requiere para satisfacer sus necesidades". 
de problemas identificados de manera aleatoria en el espacio geográfico, sino que tras la exploración de los patrones espaciales se puede identificar trampas de pobreza de tipo espacial, en donde regiones completas pueden padecer dicha condición y los esfuerzos locales difícilmente podrán ayudar a mejorar las condiciones o tendrán consecuencias limitadas. Consecuentemente, es necesario ampliar el estudio de la relevancia de la inclusión financiera y su impacto en el desarrollo económico mediante la consideración de la dimensión espacial para entender mejor las diferencias que existen entre las unidades de análisis y proponer estrategias más efectivas de política económica que atiendan los rezagos de las regiones más necesitadas.

Como una evidencia de la espacialidad de los problemas de desarrollo en los municipios de México, se presenta en la Figura 2 la distribución de la proporción de pobres a nivel municipal en el territorio nacional, en donde se observa que en el sur y sureste del país se registran elevados niveles de pobreza de manera generalizada, mientras que en el norte del país esa condición tiene un nivel más bajo. Este comportamiento guarda una relación importante con el presentado en Pérez Akaki y Fonseca (2015), quienes construyeron un índice municipal de inclusión financiera basado en la metodología Chakravarty y Pal (2010) y cuya distribución en el territorio mexicano se presenta en la Figura 3.

Figura 2. Distribución espacial de la pobreza municipal en México, 2010.

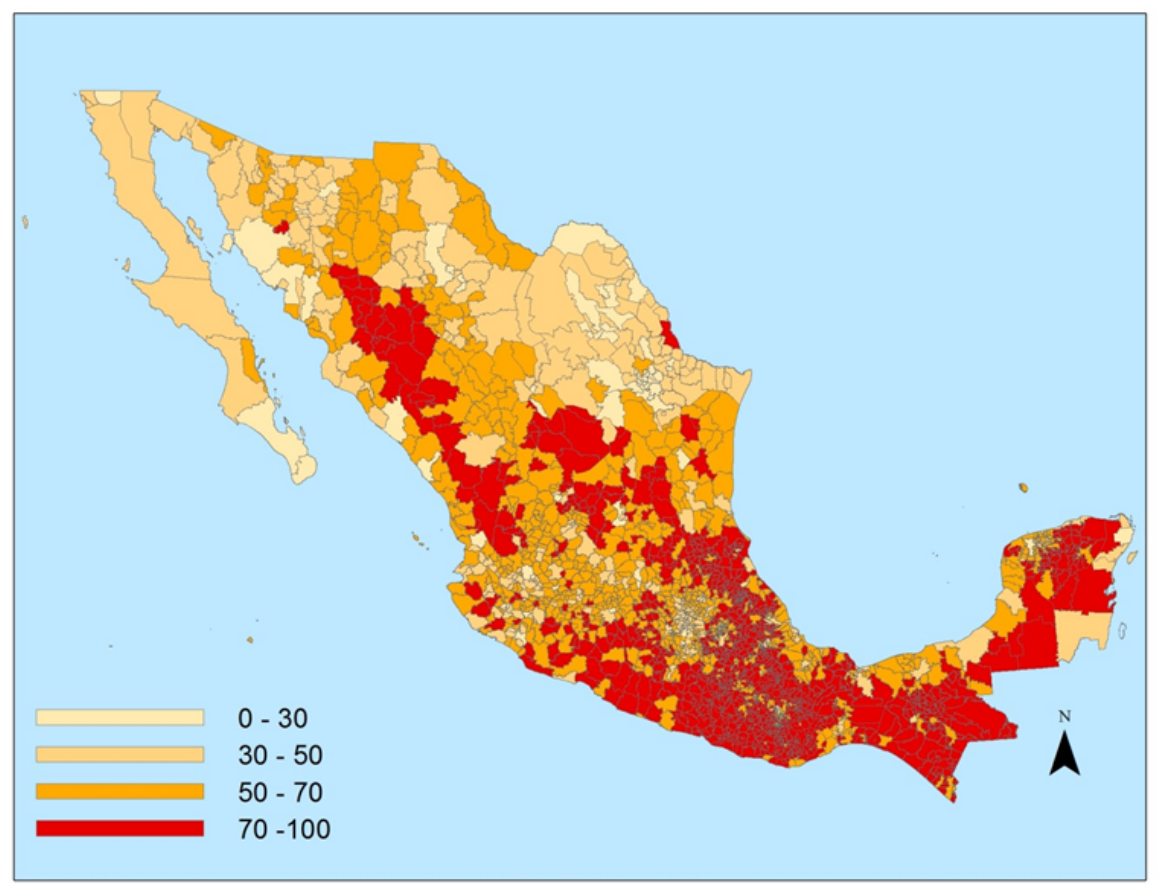

Fuente: Elaboración propia con datos de CONEVAL. 


\section{Análisis espacial de la inclusión financiera y la pobreza municipal en México}

Las teorías sobre crecimiento económico con base en el sector financiero sostienen que para salir de la pobreza es necesario aumentar los servicios y productos financieros, en combinación con otras estrategias como aumentar el nivel educativo, la oferta de servicios básicos y de salud (Lustig, 2004). Así, es posible construir un modelo empírico para estudiar la relación entre ambas variables.

Para analizar dicha relación se propuso un modelo empírico que incluye el acceso a sucursales del sector financiero como la banca comercial, la banca de desarrollo, entidades de ahorro y crédito popular y las cooperativas. Adicionalmente, como variables de control se incluyeron tasa de analfabetismo, porcentaje de población que se dedica a las actividades primarias y el nivel de ruralidad de las unidades geográficas, que en este caso corresponden a municipios. El modelo incluye variables relacionadas con el espacio como la densidad poblacional y la distancia de cada municipio respecto al municipio que representa la capital de su estado. Si bien el indicador de distancia es lineal, representa una primera aproximación al posicionamiento de cada unidad político-administrativa respecto a su referente regional (estatal). ${ }^{5}$

Figura 3. Inclusión financiera municipal en México, 2011.

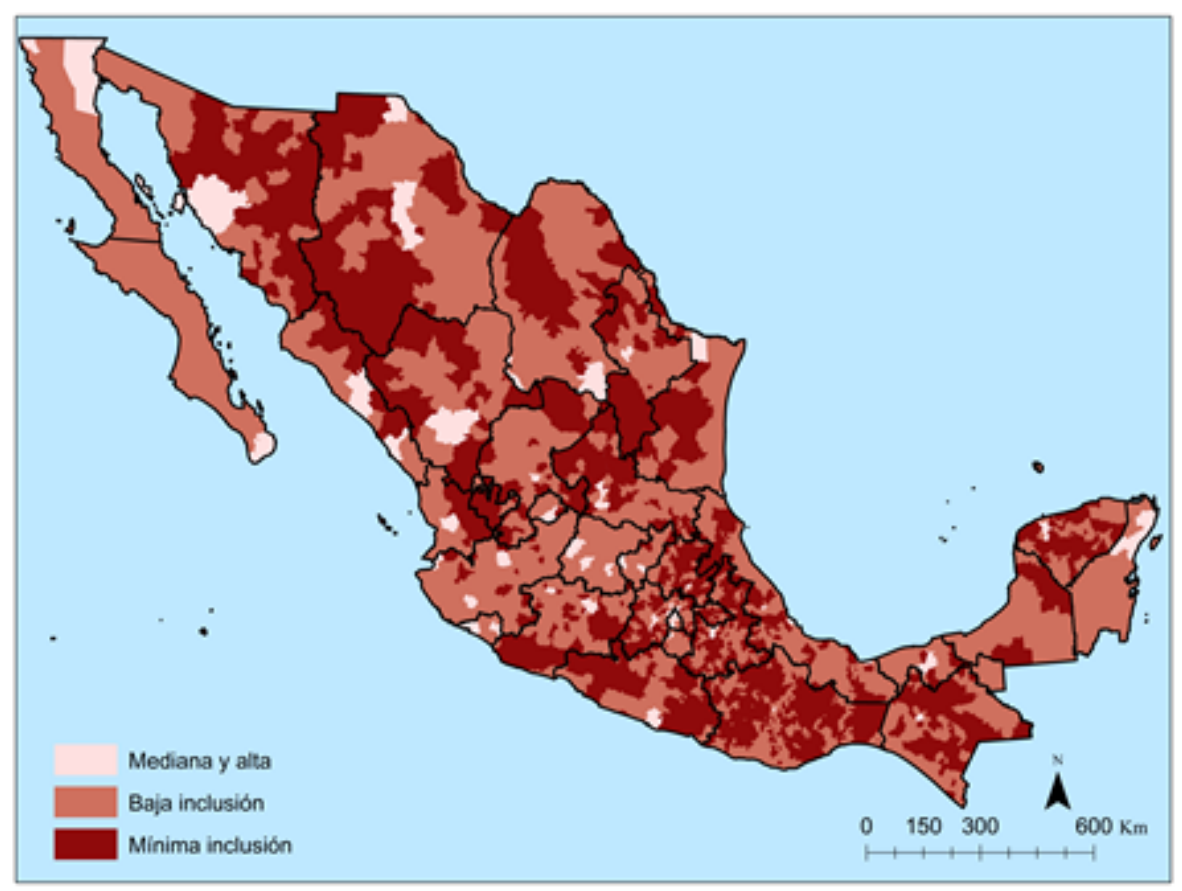

Fuente: Pérez Akaki y Fonseca, 2015.

\footnotetext{
5 Existen diversas formas de identificar distancias de los municipios, las cuales se dejan para otro trabajo posterior.
} 
En la propuesta de un modelo espacial se considera como hipótesis que la pobreza de los municipios contiguos es un factor que explica la pobreza en un municipio en particular, es decir, que se trata de un factor sistémico regional. Un análisis de la Figura 2 permite identificar que la pobreza no se distribuye aleatoriamente en los municipios del país, sino que existen regiones donde la pobreza se concentra y guarda una relación con los municipios vecinos, conformando regiones de pobreza. Para verificar dicha hipótesis se propone la medición de la I global de Moran, estadístico que mide la correlación espacial entre vecinos de un indicador para unidades territoriales. ${ }^{6}$

Para su cálculo se requiere la estimación de una matriz de pesos espaciales (también llamada de rezagos espaciales) que se representa por $\mathbf{W}$, la cual es un instrumento que registra las interdependencias entre las unidades geográficas analizadas. W es una matriz cuadrada no estocástica cuyos elementos $w_{i j}$ reflejan la intensidad de la interdependencia existente entre cada par de unidades territoriales $i$ y $j$, que tiene la siguiente forma:

$$
\left[\begin{array}{ccc}
0 & \cdots & w_{1 N} \\
\vdots & \ddots & \vdots \\
w_{N 1} & \cdots & 0
\end{array}\right]
$$

Los pesos espaciales que contiene la matriz se pueden definir de varias maneras pero en todas se requiere que dichos pesos sean finitos y no negativos. En el Cuadro 2 se muestran los criterios para la identificación de las regiones vecinas con adyacencia física.

Una de las definiciones más utilizadas es la que se refiere al concepto de contigüidad física de primer orden, donde $w_{i j}$ es igual a 1 si las regiones $i \mathrm{y}$ $j$ son físicamente adyacentes o a 0 en caso contrario (se asume por definición que $\left.w_{i i}=0\right)$. El criterio de vecindad que se utilizó en nuestro análisis fue el reina o queen. Para obtener la $I$ de Moran del porcentaje de pobres a nivel municipal en México para 2010 se utilizó el software GeoDa y su estimación global y local se muestra en la Figura 4 . El valor obtenido para la $I$ de Moran (0.681596) es significativo y positivo, lo que muestra evidencia de correlación espacial positiva municipal en la variable pobreza.

\footnotetext{
6 La hipótesis nula del estadístico I de Moran es la no correlación espacial, por lo que un valor no significativo de la prueba conduce a no rechazar dicha hipótesis; mientras que un valor significativo positivo informa acerca de la presencia de la concentración de valores similares de la variable entre regiones vecinas.
} 
Cuadro 2. Criterios de contigüidad física en una cuadricula regular.

\begin{tabular}{|l|c|l|}
\hline \multicolumn{1}{|c|}{$\begin{array}{c}\text { Criterio de } \\
\text { vecindad }\end{array}$} & $\begin{array}{c}\text { Número } \\
\text { total de } \\
\text { vecinos }\end{array}$ & \\
\hline Criterio lineal & 2 & Serán vecinas de i las regiones que comparten el lado izquierdo o derecho de $\mathrm{i}$ \\
\hline Criterio torre o rook & 4 & Serán vecinas de i las regiones que comparten algún lado con $\mathrm{i}$ \\
\hline $\begin{array}{l}\text { Criterio alfil o } \\
\text { bishop }\end{array}$ & 4 & Serán vecinas de i las regiones que comparten algún vértice con $\mathrm{i}$ \\
\hline $\begin{array}{l}\text { Criterio reina o } \\
\text { queen }\end{array}$ & 8 & Serán vecinas de i las regiones que comparten algún lado o vértice con $\mathrm{i}$ \\
\hline
\end{tabular}

Fuente: Moreno y Vayá, 2000.

La expresión territorial de la correlación municipal de la pobreza en México permite la identificación de agrupaciones regionales (clusters) de alta y baja pobreza, así como combinaciones de alta-baja y baja-alta pobreza. El estadístico que permite dicha medición espacial es la $I i$ de Moran ( $I$ local de Moran), la cual se presenta en la Figura 4 donde se confirma que la pobreza municipal no está distribuida de forma aleatoria en el territorio mexicano, sino que forma regiones, lo que sugiere que las políticas que la atiendan deberían considerar esa concentración espacial.

Previamente Pérez Akaki y Fonseca (2015) habían identificado la concentración espacial de la inclusión financiera en México, además de la existencia de una relación espacial entre inclusión financiera municipal y pobreza de los municipios vecinos. Sin embargo, no se trata de un modelo explicativo, sino de una correlación estadística en donde se incorpora el espacio, por lo que cuando quiere cuantificarse una relación causal entre la inclusión financiera y la pobreza en el análisis territorial, es necesario construir un modelo de regresión espacial. Entre ellos se encuentran el modelo de rezago espacial (spatial lag) y el modelo de error espacial (spatial error), en los cuales es necesario trabajar con la matriz de vecindades $\mathbf{W}$ que permite incorporar las influencias mutuas entre las unidades espaciales. Respecto de las pruebas para contrastar las estructuras de dichos modelos Anselin y Rey (Moreno y Vayá, 2000) concluyen que las basadas en el principio de multiplicadores de Lagrange son las mejores para detectar el esquema adecuado de dependencia espacial presente en los datos. 
Figura 4. Concentración espacial de la pobreza municipal en México, 2010 I global de Morán: 0.681596.

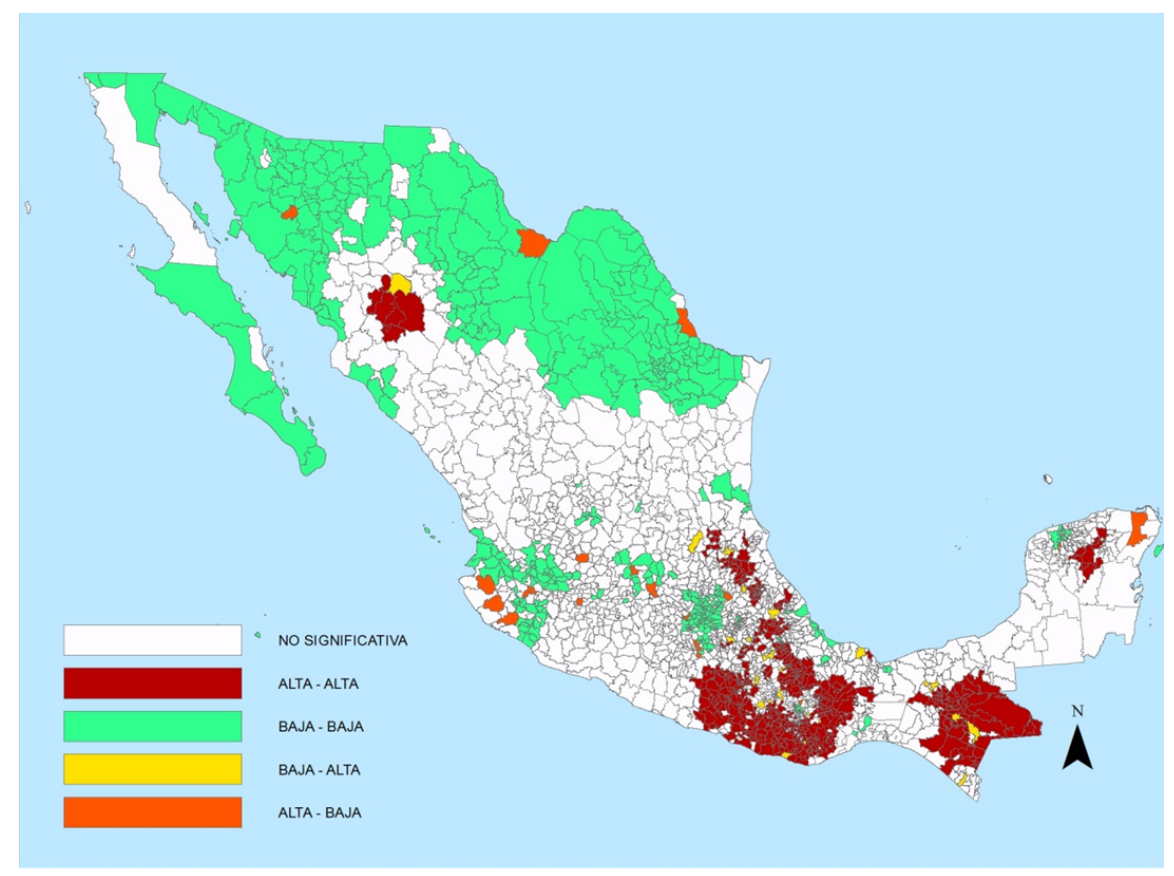

Fuente: Elaboración propia con datos de CONEVAL.

5. Regresión espacial para la pobreza municipal y la inclusión financiera

A continuación se estiman los dos modelos clásicos de regresión espacial, rezagos y errores espaciales, los cuales se compararán con el modelo clásico de regresión lineal. Los modelos especiales pueden identificarse con las ecuaciones siguientes:

$$
\begin{gathered}
y=\rho W y+X \beta+\varepsilon \\
\varepsilon=\lambda W \varepsilon+u \\
u\left(0, \sigma^{2} I\right)
\end{gathered}
$$

donde $\rho$ representa el coeficiente de rezago espacial, $\lambda$ el coeficiente de error espacial y $W$ la matriz de pesos espaciales discutida previamente. En el caso del modelo de rezago espacial, le corresponde $\lambda=0$ mientras que para el modelo con errores espaciales el coeficiente $\rho=0^{7}$

La matriz de variables independientes $(X)$ en el modelo incorpora los siguientes indicadores:

\footnotetext{
7 Al modelo en donde ambos parámetros son diferentes de cero se le conoce como de rezagos y errores espaciales (SARMA en inglés), el cual será tema de análisis en otro trabajo.
} 
a) Densidad poblacional (Habitantes por kilómetro cuadrado): La relación entre un espacio determinado y el número de personas que lo habita se llama densidad de población, la cual se obtiene dividiendo el número de personas que viven en un lugar específico entre el número de kilómetros cuadrados que mide ese territorio. En nuestro país la densidad poblacional es baja en la mayoría de los municipios del país y solamente en los municipios del centro es mayor.

b) Tasa de analfabetismo: Es un indicador que refleja los rezagos educativos en un país. Se calcula a partir de la población de 15 años y más que no sabe leer y escribir dividido por la población de 15 años y más, multiplicado por 100. En el norte del país el analfabetismo es bajo, excepto para algunos municipios de Chihuahua.

c) Población ocupada en el sector primario (porcentaje del total poblacional): Se refiere a la población ocupada en el sector primario (agricultura, ganadería, silvicultura, caza y pesca) para cada municipio de acuerdo con el Censo de Población y Vivienda 2010; los municipios con más del $75 \%$ de su población ocupada en actividades primarias se ubican mayoritariamente hacia el sur.

d) Ruralidad: La medición de ruralidad se realiza identificando la proporción de personas que radican en el municipio en localidades menores a 15 mil habitantes respecto al total poblacional. De esta manera se construye una variable continua que da cuenta del grado de fragmentación poblacional en el municipio que se usa como proxi para la ruralidad del municipio. ${ }^{8}$

e) Distancias: La distancia respecto al centro económico regional es un elemento de gran importancia para explicar las diferencias que existen en el territorio en cuanto a su nivel de desarrollo, siendo esta una variable que aproxima a la marginación de los municipios. La distancia se midió en kilómetros en línea recta desde la cabecera de la entidad hasta cada municipio. ${ }^{9}$

f) Inclusión financiera: Tomando como referencia los resultados en Pérez Akaki y Fonseca (2015) se utiliza el índice de inclusión financiera municipal como variable explicativa en el modelo de regresión de la pobreza municipal en México. Adicionalmente se descompone el índice mencionado en dos subíndices, inclusión financiera de acceso e inclusión financiera de uso, para destacar mejor la relevancia de la inclusión financiera como indicador de profundidad.

Los resultados del modelo estimado se presentan en el Cuadro 3, donde se presentan 6 regresiones, 3 que utilizan la inclusión financiera total y 3 regresiones adicionales en las que la inclusión financiera se presenta por medio de los subíndices de acceso y uso. Adicionalmente se comparan en cada caso los modelos de regresión simple, errores espaciales y rezago espacial. Debido a

\footnotetext{
8 Este criterio cuestiona la definición de INEGI de usar un total criterio poblacional de 2,500 para definir ruralidad, sin embargo no lo hace de manera tajante al crear dos categorías (rural-urbano) sino que se expresa como proporción del total poblacional del municipio.

9 La medición se hizo calculando los centroides de cada municipio y posteriormente su distancia respecto al centroide del municipio que aloja la cabecera de la entidad. Las distancias se calcularon en línea recta, sin considerar el relieve lo cual será un criterio para ajustarse en futuros trabajos. Cada municipio se compara contra su propio centro estatal, sin considerar si hubiera otro centro de menor distancia correspondiente a otra entidad.
} 
que sólo se mantuvieron en el cuadro las variables relevantes, existen espacios en blanco en algunas de ellas, mientras que en los coeficientes que si aparecen se tiene un nivel de significancia superior al $99 \%$.

Cuadro 3. Resultados de los modelos de regresión: inclusión financiera y pobreza.

\begin{tabular}{|c|c|c|c|c|c|c|}
\hline Variables & $\begin{array}{l}\text { Mínimos } \\
\text { cuadrados } \\
\text { ordinarios }\end{array}$ & $\begin{array}{c}\text { Modelo de } \\
\text { error esp acial }\end{array}$ & $\begin{array}{c}\text { Modelo de } \\
\text { rezago } \\
\text { espacial }\end{array}$ & $\begin{array}{l}\text { Mínimos } \\
\text { cuadrados } \\
\text { ordinarios }\end{array}$ & $\begin{array}{c}\text { Modelo de } \\
\text { error esp acial }\end{array}$ & $\begin{array}{c}\text { Modelo de } \\
\text { rezago } \\
\text { espacial }\end{array}$ \\
\hline \multirow{2}{*}{ Constante } & 43.11896 & 47.63596 & 19.66770 & 47.68140 & 48.20386 & 22.35064 \\
\hline & $(1.19421)$ & $(1.18883)$ & $(1.25113)$ & $(1.23676)$ & $(1.20542)$ & $(1.22511)$ \\
\hline \multirow{2}{*}{ Tasa analfabetismo } & 0.86049 & 0.68295 & 0.45558 & 0.80801 & 0.68145 & 0.44438 \\
\hline & $(0.02770)$ & $(0.03291)$ & $(0.02632)$ & $(0.02734)$ & $(0.03279)$ & $(0.02640)$ \\
\hline \multirow{2}{*}{$\begin{array}{l}\text { Proporción sector } \\
\text { primario }\end{array}$} & 0.29408 & 0.25770 & 0.21059 & 0.26680 & 0.25099 & 0.20061 \\
\hline & $(0.01307)$ & $(0.01292)$ & $(0.01131)$ & $(0.01295)$ & $(0.01314)$ & $(0.01131)$ \\
\hline \multirow{2}{*}{ Ruralidad } & 4.83102 & 2.65016 & 4.76327 & 2.43101 & 2.43759 & 4.01299 \\
\hline & $(0.98865)$ & $(0.879904)$ & $(0.80548)$ & $(0.98532)$ & $(0.80362)$ & $(0.77890)$ \\
\hline \multirow{2}{*}{$\begin{array}{c}\text { Densidad } \\
\text { poblacional }\end{array}$} & -0.00057 & -0.00069 & & -0.00092 & -0.00080 & \\
\hline & $(0.00019)$ & $(0.00027)$ & & $(0.00019)$ & $(0.00027)$ & \\
\hline \multirow{2}{*}{$\begin{array}{c}\text { Distancia a capital } \\
\text { estatal }\end{array}$} & -0.00002 & & -0.00003 & -0.00002 & & -0.00002 \\
\hline & $(0.00001)$ & & $(0.00000)$ & $(0.00001)$ & & $(0.00000)$ \\
\hline \multirow{2}{*}{ Inclusión financiera } & -22.72827 & -25.50216 & -23.14262 & & & \\
\hline & $(3.70003)$ & $(3.53016)$ & $(3.02905)$ & & & \\
\hline \multirow{2}{*}{$\begin{array}{c}\text { Inclusión } \\
\text { financiera-acceso }\end{array}$} & & & & -45.11099 & -12.04869 & -26.81167 \\
\hline & & & & $(3.73232)$ & $(3.65846)$ & $(2.55370)$ \\
\hline \multirow{2}{*}{$\begin{array}{c}\text { Inclusión } \\
\text { financiera-uso }\end{array}$} & & & & 12.06765 & -14.10485 & \\
\hline & & & & $(4.36238)$ & $(4.70009)$ & \\
\hline \multirow{2}{*}{$\begin{array}{c}\text { Error espacial } \\
(\lambda)\end{array}$} & & 0.69343 & & & 0.68353 & \\
\hline & & $(0.01819)$ & & & $(0.01853)$ & \\
\hline \multirow{2}{*}{ Rezago espacial ( $\rho)$} & & & 0.48736 & & & 0.46608 \\
\hline & & & $(0.01584)$ & & & $(0.01593)$ \\
\hline $\begin{array}{c}\text { Razón de } \\
\text { verosimilitud }\end{array}$ & -9125.35 & -8667.620055 & -8725.49 & -9058.84 & -8663.696542 & -8700.82 \\
\hline Criterio de Akaike & 18264.70 & 17347.20 & 17465.00 & 18133.70 & 17341.40 & 17415.60 \\
\hline Criterio de Schwarz & 18305.30 & 17382.10 & 17505.60 & 18180.10 & 17382.00 & 17456.30 \\
\hline $\mathrm{R}^{2}$ & 0.710801 & 0.821909 & 0.801157 & 0.726054 & 0.821772 & 0.804210 \\
\hline
\end{tabular}

Nota: Los números en paréntesis representan la desviación estándar del estimador.

Fuente: Elaboración propia.

Como puede observarse en el Cuadro 3, todos los modelos estimados a escala municipal sobre el territorio nacional muestran una alta significancia. Ésta mejora sustancialmente al introducir los modelos espaciales y ligeramente al separar la inclusión financiera en sus componentes. Adicionalmente, los modelos de errores espaciales superan ligeramente a los de rezago espacial, evidenciando que existen algunos otros elementos no incluidos en las regresiones que explican el comportamiento de la pobreza en el territorio nacional.

Dada la relevancia estadística de los rezagos espaciales, es importante destacar el efecto que ejerce en la pobreza de un municipio la pobreza de los vecinos. Ello implica que aun cuando se realicen inversiones de 
importancia para combatir la pobreza en algunos de ellos, existen factores implícitos inherentes a un conjunto de municipios que comparten vecindad que deberán ser identificados y resueltos para que las estrategias de combate a la pobreza y desarrollo económico tengan mejores resultados.

El análisis de las variables de control en el modelo evidenció que los signos son los esperados: positivo para el caso de analfabetismo, ruralidad y actividades primarias y negativo para densidad y distancia. Adicionalmente, en todos los casos se identificó una relación negativa importante entre la inclusión financiera y la pobreza de los municipios mexicanos, en los cuales se tiene signo negativo con alta significancia (mayor a 99\%), tanto en los modelos espaciales como en el modelo clásico.

Entre los subíndices de acceso y uso de la inclusión financiera, resultó ser de mayor utilidad para disminuir la pobreza el de acceso, pues en el análisis el subíndice de uso resultó positivo en el caso de la estimación de mínimos cuadrados ordinarios (MCO) y no resultó significativo en el caso del modelo de rezagos espaciales, mientras que en el modelo de errores espaciales tuvo el signo esperado (negativo) y fue estadísticamente significativo.

Para comprender el comportamiento de las variables de inclusión financiera (acceso y uso), pobreza municipal y su distribución espacial, en la Figura 5 se presenta un mapa condicional que las vincula y permite apreciar su patrón de comportamiento.

Figura 5. Mapa condicional de pobreza e inclusión financiera uso y acceso.

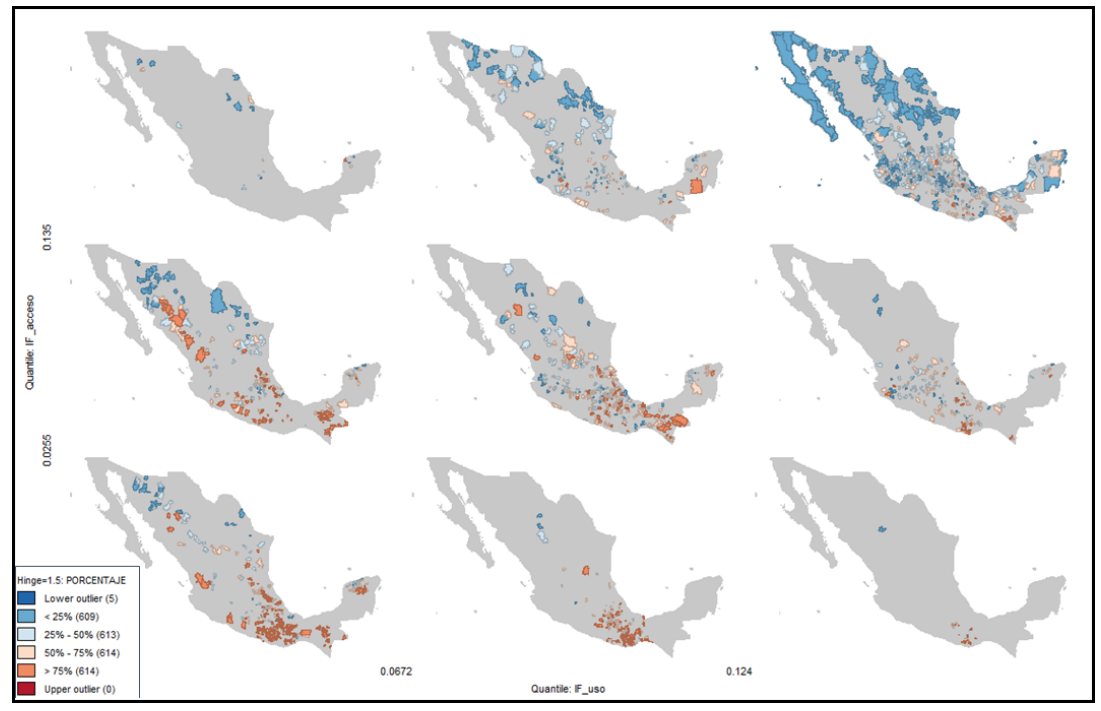

Fuente: Elaboración propia.

Las tonalidades azules representan niveles bajos de pobreza, mientras que los rojos representan altos niveles de pobreza municipal. El eje X representa a la variable Inclusión financiera de uso, mientras que el eje Y representa la variable Inclusión financiera de acceso. Cada columna y reglón esta graduada, de tal 
manera que podría decirse que se identifican niveles bajos, medios y altos de inclusión financiera por uso en las columnas y por acceso en los renglones. ${ }^{10}$

Claramente, los niveles altos de pobreza se identifican preferentemente en los municipios del sur y sureste de México, los cuales intersectan con niveles bajos de inclusión financiera de acceso y uso. Este resultado confirma los resultados encontrados en el modelo econométrico e ilustra la manera en la que se organizan los municipios según este conjunto de variables de interés.

Finalmente, se destaca que en los modelos espaciales, tanto los parámetros de rezagos como de errores espaciales resultaron relevantes y con los signos esperados, mejorando éstos últimos el nivel de ajuste del modelo de regresión en todos los casos. La pobreza de los municipios vecinos es una característica relevante para entender la pobreza de cada municipio además que el error espacial sugiere la presencia de otras variables espaciales necesarias para explicar mejor esta variable dependiente

\section{Conclusiones}

El sistema financiero puede tener gran relevancia para impulsar el crecimiento económico de los países, así como para explicar las diferencias en el desarrollo entre regiones al interior de un país. Las funciones que desde la teoría se han identificado serían igualmente valiosas en los entornos urbanos y rurales, así como en las regiones de especialización en actividades primarias, secundarias y terciarias. Sin embargo, desde la economía regional se sabe que existen diferencias importantes entre las regiones, lo cual puede resultar en comportamientos diferentes, a veces incluso contrarios a lo predicho teóricamente.

Con herramientas de análisis estadístico espacial, en este trabajo se exploró sobre la relevancia de la inclusión financiera sobre la pobreza municipal, en un esfuerzo por entender de mejor manera la relación entre estas variables cuando el espacio se vuelve una variable explícita en el análisis. Los resultados mostraron que es importante incluir dicha dimensión en el análisis, por lo que es necesario concebir de manera más amplia, en términos de territorio, las soluciones que a dichas problemáticas puedan diseñarse.

Se identificó además que la inclusión financiera es relevante, pero no tanto cuando se construyeron subíndices de uso y acceso. La expectativa es que el uso de instrumentos financieros tuviera una relación más relevante con la reducción de la pobreza, pero no pudo sostenerse en el análisis de los municipios del país. En parte este resultado negativo sobre el uso podría explicarse por la deficiencia en la medición del uso, que está relacionado con el número de contratos, no con las transacciones realizadas por ejemplo, lo que sería una medida más apropiada. Por el contrario, resultó relevante la presencia de sucursales, corresponsales y puntos de venta (subíndice de acceso).

El ejercicio puede extenderse incluyendo mediciones más recientes para poder cuantificar el dinamismo del sector financiero y su influencia para mejorar las condiciones de vida de la sociedad mexicana. Igualmente, es necesario contar con mayores detalles sobre la actividad financiera que permitan medir de mejor manera su impacto en la economía del país.

\footnotetext{
10 Cada indicador de inclusión financiera en los ejes se dividió en 3 cuantiles, quedando entonces dividida la escala total de valores en percentiles al $33 \%$.
} 


\section{Bibliografía}

Akaki, P. \& Fonseca, S. (2015). La inclusión financiera en los municipios mexicanos en los comienzos del siglo XXI. En Pérez Lechuga, G., F. Venegas Martínez y J. F. Martínez Sánchez. Modelos para la toma de decisiones en la ingeniería económica y financiera: un enfoque estocástico. 2 ed. México: Universidad Autónoma del Estado de Hidalgo e Instituto Politécnico Nacional.

Ang, J. (2008). A Survey of Recent Developments in the Literature of Finance and Growth. Journal of Economic Surveys, 22(3), 536-576.

Anselin, L. (2005). Exploring Spatial Data with GeoDaTM: A Workbook. Center for Spatially Integrated Social Science.

Anselin, L. (1988). Spatial Econometrics: Methods and Models. Kuwler Academic Publishers, The Netherlands.

Avalos, M. y Hernández, T. (2006). Competencia bancaria en México. Serie Estudios y Perspectivas 62, CEPAL, México.

Beck, T., A. Demirguc-Kunt, \& R. Levine. (2004). Finance, Inequality and Poverty: Cross-Country Evidence. NBER, Working Paper 10979.

Center for Financial Inclusion - CFI. (2009a). Financial Inclusion: What's the vision?. Center for Financial Inclusion.

Center for Financial Inclusion - CFI.(2009b). Mexico's Prospects for Full Financial Inclusion. Center for Financial Inclusion.

Claessens, S. \& E. Feijen. (2006). Financial Sector Development and the Millennium Development Goals. World Bank Working Paper, 89.

Comisión Nacional Bancaria y de Valores (2011). Reporte de Inclusión Financiera 3. México.

Comisión Nacional Bancaria y de Valores (2012). Reporte de Inclusión Financiera 4. México.

Demirguc-Kunt, A. \& R. Levine. (2001). Financial Structure and Economic Growth: Perspectives and Lessons, en Demirguc-Kunt, A. y R. Levine. Financial Structure and Economic Growth. A Cross-Country Comparison of Banks, Markets, and Development, pp. 3-14.

Demirguc-Kunt, A. y Klapper, L. (2012). Measuring Financial Inclusion: The Global Findex Database. The World Bank.

Diario Oficial de la Federación (03/10/2011). Acuerdo por el que se crea el Consejo Nacional de Inclusión Financiera.

Goldsmith, R. (1963). La estructura financiera y el crecimiento económico. CEMLA, México.

Honohan, P. (2004). Financial Development, Growth and Poverty: How Close are the Links? World Bank Policy Research Working Paper 3203.

Instituto Nacional de Estadística y Geografía (2012). Encuesta Nacional de Inclusión Financiera. Análisis descriptivo de los resultados

Khalifa, Y. (2002). Financial Development and Economic Growth. Another Look at the Evidence from Developing Countries. Review of Financial Economics, 11, 131-150.

La Porta, R. et al. (1997). Legal Determinants of External Finance. The Journal of Finance $52(3), 1131-1150$.

Levine, R. (1997). Financial Development and Economic Growth: Views and Agenda. Journal of Economic Literature 35 (2), 688-726.

Levine, R. y King, R. (1993). Finance and Growth: Schumpeter might be Right. The Quarterly Journal of Economics 108 (3), 717-736.

Lustig, N. (2004). Crecimiento económico y reducción de la pobreza en Boltvinic, J. y A. Damián. La pobreza en México y el mundo. México: Siglo XXI editores.

Moreno, R. \& Vayá V. (2000). Técnicas Econométricas para el tratamiento de datos espaciales: la econometría espacial. Barcelona, España: Edicions Universitat.

Comisión Nacional Bancaria y de Valores. Consultado en: http://www.cnbv.gob.mx/

Consejo Nacional de Evaluación de la Política de Desarrollo Social. Consultado en: http:// www.coneval.gob.mx/

Instituto Nacional de Estadística y Geografía. Consultado en: http://www.inegi.org.mx/

Global Findex (Global Financial Inclusion Database). Consultado en: http://databank. worldbank.org/data/views/variableselection/selectvariables.aspx?source=global-findex 
62 Nueva Época REMEF (The Mexican Journal of Economics and Finance)

Peña, P. \& Vázquez, A. (2012). El impacto de los corresponsables bancarios en la inclusión financiera: una primera evaluación. Estudios Económicos CNVB (1), 195-215.

Rivera, E. \& Rodríguez, A. (2007). Competencia y regulación de la banca de Centroamérica y México. Un estudio comparativo. Serie Estudios y Perspectivas 71, México: CEPAL.

Rodríguez, D. y López, F. (2009). Desarrollo financiero y crecimiento económico en México. Problemas del desarrollo 40 (159), 39-60.

Tobler, W. (1970). A Computer Movie Simulating Urban Growth in the Detroit Region. Economic Geography 46, 234-240. 